Radiologe $2020 \cdot 60: 711-720$

https://doi.org/10.1007/s00117-020-00726-x

Online publiziert: 24 . Juli 2020

(c) Der/die Autor(en) 2020
Hannah Schneider ${ }^{1}$ Anne-Lena Illert ${ }^{2}$. Fabian Bamberg ${ }^{1} \cdot$ Michel Eisenblätter $^{1}$

' Klinik für Diagnostische und Interventionelle Radiologie, Universitätsklinikum Freiburg, Freiburg, Deutschland

${ }^{2}$ Klinik für Innere Medizin I - Onkologie, Universitätsklinikum Freiburg, Freiburg, Deutschland

\title{
Monitoring von Immuntherapien
}

\section{Möglichkeiten und Grenzen}

Mit der Etablierung von Immuntherapien in der Onkologie haben sich neue Ansprüche an die bildgebende Diagnostik ergeben. Für eine präzise Befundung und Beurteilung der Therapieverläufe und der $\mathrm{Ne}$ benwirkungen sind eine Kenntnis der Erscheinungsformen, Inzidenzen und zeitlichen Verläufe sowie eine einheitliche Nomenklatur und standardisierte Evaluationskriterien notwendig. In diesem Übersichtsartikel soll aufgezeigt werden, was es im radiologischen Monitoring von Immuntherapien im Hinblick auf Therapieverlauf und Nebenwirkungen zu beachten gilt und wie aktuelle Probleme angegangen werden können.

\section{Überblick Immuntherapien}

Als Immuntherapeutika werden in der Onkologie Substanzen bezeichnet, die ihre antitumorale Wirkung über eine Immunreaktion entfalten. Diese kann passiver oder aktiver Natur sein. Passive Immuntherapie bedeutet hierbei die direkte Applikation antineoplastisch wirkender Antikörper - z. B. der Anti-CD20Antikörper Rituximab zur Therapie bei B-Zell-Non-Hodgkin-Lymphomen oder Trastuzumab gegen Her2-Rezeptor-exprimierende Tumoren - auf welche in diesem Artikel nicht näher eingegangen werden soll.

Aktive Immuntherapeutika stimulieren eine körpereigene Abwehrreaktion gegenüber Tumorzellen. Hierbei sind vor allem die sog. Checkpoint-Inhibitoren von radiologischem Interesse. Als Immuncheckpoints werden immunmodulierende, zumeist inhibitorische
Signalwege bezeichnet, über welche Immunzellen kontrolliert und überschieBende oder autoimmune Reaktionen verhindert werden [34, 47]. Eine der von Tumorzellen genutzten Möglichkeiten zur Unterwanderung des Immunsystems ist die Imitation dieser Checkpoints, wodurch die Tumorabwehr inhibiert wird [47]. Von therapeutischem Nutzen sind bisher die zu dieser Klasse zählenden, auf T-Zellen exprimierten Rezeptoren CTLA-4 („cytotoxic T-lymphocyteassociated protein $\left.4^{\prime \prime}\right)$ und PD-1 („programmed cell death protein-1"). Auch der Ligand des PD-1-Rezeptors PD-L1, welcher von Stromazellen oder eben von Tumorzellen exprimiert wird, stellt einen pharmazeutischen Angriffspunkt dar.
Diese Rezeptoren bzw. der Ligand können mittels monoklonaler Antikörper blockiert werden, wodurch die Inhibition antitumoraler Wirkungen vermindert und eine physiologische, T-Zell-vermittelte Destruktion neoplastischer Zellen ermöglicht wird [34]. Anders als bei klassischen Chemotherapeutika wirken Immuntherapeutika also nicht durch eine toxische Destruktion von Tumorzellen, sondern durch eine Stärkung der körpereigenen Immunreaktion.

Als erster Vertreter dieser neuen Substanzen wurde der CTLA-4-Antikörper Ipilimumab im Jahr 2011 zunächst zur Behandlung des metastasierten malignen Melanoms zugelassen. Einen Überblick über weitere etablierte Immun-Check-
Tab. 1 Zugelassene Immun-Checkpoint-Inhibitoren mit jeweiligen Indikationen

\begin{tabular}{|l|l|l|l|}
\hline $\begin{array}{l}\text { Angriffs- } \\
\text { punkt }\end{array}$ & Wirkstoff & $\begin{array}{l}\text { Zulassung } \\
\text { (EU) }\end{array}$ & Indikation (Stand 01/20) \\
\hline CTLA-4 & Ipiliumab & $12 / 13$ & $\begin{array}{l}\text { Melanom Mono oder Kombi mit Nivolumab } \\
\text { NCC Kombi mit Nivolumab }\end{array}$ \\
\hline PD-1 & Pembrolizumab & $10 / 16$ & $\begin{array}{l}\text { Melanom Mono } \\
\text { NSCLC Mono oder Kombi mit Chemotherapie }\end{array}$ \\
& & & $\begin{array}{l}\text { Hodgkin-Lymphom Mono } \\
\text { Urothelkarzinom Mono }\end{array}$ \\
& & & $\begin{array}{l}\text { HNSCC Mono oder Kombi mit Chemotherapie } \\
\text { NCC Kombination mit Axitinib }\end{array}$ \\
\hline & Nivolumab & $04 / 16$ & $\begin{array}{l}\text { NSCLC } \\
\text { Melanom Mono oder Kombi mit Ipilimumab }\end{array}$ \\
& & & NCC Kombi mit Ipilimumab \\
\hline PD-L1 & Atezolizumab & $09 / 17$ & Urothelkarzinom Mono \\
& & NSCLC Mono \\
\hline & Durvalumab & $09 / 18$ & NSCLC \\
\hline Avelumab & $10 / 17$ & Merkel-Zell-Karzinom \\
\hline
\end{tabular}

CTLA-4 „Cytotoxic T-lymphocyte antigen-4", PD-1 „programmed cell death protein-1“, PD-L1 „programmed cell death protein-1 ligand-1", Mono Monotherapie, Kombi Kombinationstherapie, NCC Nierenzellkarzinom, NSCLC nichtkleinzelliges Lungenkarzinom, HNSCC Plattenepithelkarzinom der Kopf-Hals-Region 
point-Inhibitoren und ihre Indikationen gibt $\bullet$ Tab. 1. Neben der Behandlung fortgeschrittener Krebserkrankungen nach Versagen anderer Therapieoptionen können unter bestimmten Voraussetzungen Immuntherapeutika auch in der Erstlinientherapie eingesetzt werden, beispielsweise bei PD-1-positiven nichtkleinzelligen Lungenkarzinomen (NSCLC; [23]). Die Ansprechraten der Immuntherapien variieren je nach spezifischem Therapeutikum und Tumorentität, aber auch in Abhängigkeit von den studienspezifischen Evaluationskriterien; für NSCLC liegen sie bei 10-24\% unter Pembrolizumab, für Melanome bei $28-44 \%$ unter Pembrolizumab bzw. 10-11\% unter Ipilimumab [7, 16, 21, 25, 27, 33, 37]. Insgesamt konnte ein therapeutischer Nutzen vor allem hinsichtlich einer verlängerten Überlebenszeit bei Patienten mit fortgeschrittenen Tumorstadien gezeigt werden $[33$, 37]. Trotzdem sollte erwähnt werden, dass auch unter Immuntherapie der größte Anteil (je nach Tumorentität 60-90\%) an Patienten einen weiteren Tumorprogress erleidet [16, 21]. Mit Blick auf die zu erwartende Ausweitung des Indikationsspektrums, die Entwicklung neuer Immuntherapeutika sowie die Etablierung von Kombinationstherapien wird die Kenntnis spezifischer immuntherapieassoziierter radiologischer Veränderungen in der klinischen Praxis rasch an Bedeutung gewinnen.

\section{Typisches und atypisches Therapieansprechen}

Bereits in den ersten Studien vor Marktzulassung von Ipilimumab wurde festgestellt, dass sich die Reaktionsmuster der behandelten Malignome von den Reaktionen auf eine zytostatische Chemotherapie unterscheiden [46]. Während bei klassischen Chemotherapien ein Therapieansprechen durch eine rasche Reduktion der Tumorlast charakterisiert ist, können Immuntherapien hiervon abweichende, atypische Verläufe zeigen. In klinischen Studien konnten vier verschiedene Muster eines Therapieansprechens identifiziert werden (relative Häufigkeiten exemplarisch für Patienten mit Therapieansprechen unter

Radiologe 2020 - 60:711-720 https://doi.org/10.1007/s00117-020-00726-x

(c) Der/die Autor(en) 2020

\section{H. Schneider · A.-L. Illert · F. Bamberg $\cdot$ M. Eisenblätter}

\section{Monitoring von Immuntherapien. Möglichkeiten und Grenzen}

\section{Zusammenfassung}

Hintergrund. Immuntherapien spielen in der Behandlung fortgeschrittener onkologischer Erkrankungen eine zunehmende Rolle. Bei einigen Patienten birgt die radiologische Diagnostik durch atypische, immuntherapieinduziete Therapieverläufe neue Herausforderungen.

Ziel der Arbeit. Dieser Beitrag soll einen Überblick über die bildgebenden Methoden des Monitorings von Immuntherapien geben, die assoziierten Phänomene Pseudoprogress und Hyperprogress erörtern sowie die Evaluationskriterien iRECIST vorstellen, welche sich als Evaluationsstandard für klinische Studien anbieten. Zusätzlich werden die radiologisch wichtigsten Nebenwirkungen und ihre bildmorphologischen Charakteristika beschrieben.

Material und Methoden. Für diesen Übersichtsartikel wurden Studienergebnisse und Reviews seit 2009 ausgewertet. Die Literaturrecherche erfolgte mittels PubMed, die Suchbegriffe enthielten "immunotherapy", "checkpoint inhibitor", ,"pseudoprogression", "iRECIST" und „immune related adverse events".
Ergebnisse und Diskussion. Mit einer Inzidenz von bis zu $10 \%$ ist der Pseudoprogress insgesamt selten; aktuell ist die Differenzierung von einem echten Progress nur durch eine Beobachtung des zeitlichen Verlaufs möglich. Die 2017 erschienenen iRECIST-Kriterien enthalten daher die neuen Kategorien unbestätigter (immune unconfirmed progressive disease iUPD) und bestätigter Progress (immune confirmed progressive disease iCPD). Bisher konnte keine evidenzbasierte Empfehlung bezüglich des Zeitintervalls zwischen den Untersuchungen gegeben werden. Als radiologisch wichtigste Nebenwirkungen sind die Hypophysitis und die Pneumonitis zu nennen. Letztere kann sich in verschiedenen Mustern der interstitiellen Pneumonie präsentieren. Die Differenzierung zwischen Pneumonitis, Infektion und Tumorprogress kann diagnostische Schwierigkeiten mit sich bringen.

\section{Schlüsselwörter}

Neoplasien · Checkpoint-Inhibitoren . Pseudoprogress · iRECIST - Pneumonitis

\section{Monitoring of immunotherapy. Possibilities and limitations}

\section{Abstract}

Background. Cancer immunotherapies play an increasing role in the treatment of advanced cancer. In a subset of patients, atypical response patterns and unconventional adverse events make diagnostic evaluation challenging for radiologists.

Objectives. In this article, we provide a review of the possibilities and limitations of imaging methods in monitoring immunotherapies, discuss the phenomena of pseudoprogression and hyperprogression, and introduce iRECIST as an evaluation standard for clinical studies with immunotherapies. In addition, we describe the most notable adverse events and their imaging features.

Materials and methods. This article is based on reviews and studies published since 2009. We used PubMed for the literature search and included the following search terms: "immunotherapy", "checkpoint inhibitor", "pseudoprogression", "iRECIST" and "immune related adverse events".
Results and conclusion. With an incidence of up to $10 \%$, pseudoprogression is a rare phenomenon. Currently, differentiation between pseudoprogression and true progressive disease is only possible by followup examinations. iRECIST, published in 2017, introduced immune unconfirmed progressive disease (iUPD) and immune confirmed progressive disease (iCPD) as new categories of therapeutic response. There is still no consensus on the time interval between examinations. Crucial adverse events include hypophysitis and pneumonitis, whereby the latter may present as different patterns of interstitial pneumonia making it difficult to differentiate between drug toxicity, infection, and tumor progression.

Keywords

Neoplasms · Checkpoint inhibitors .

Pseudoprogression · iRECIST · Pneumonitis 

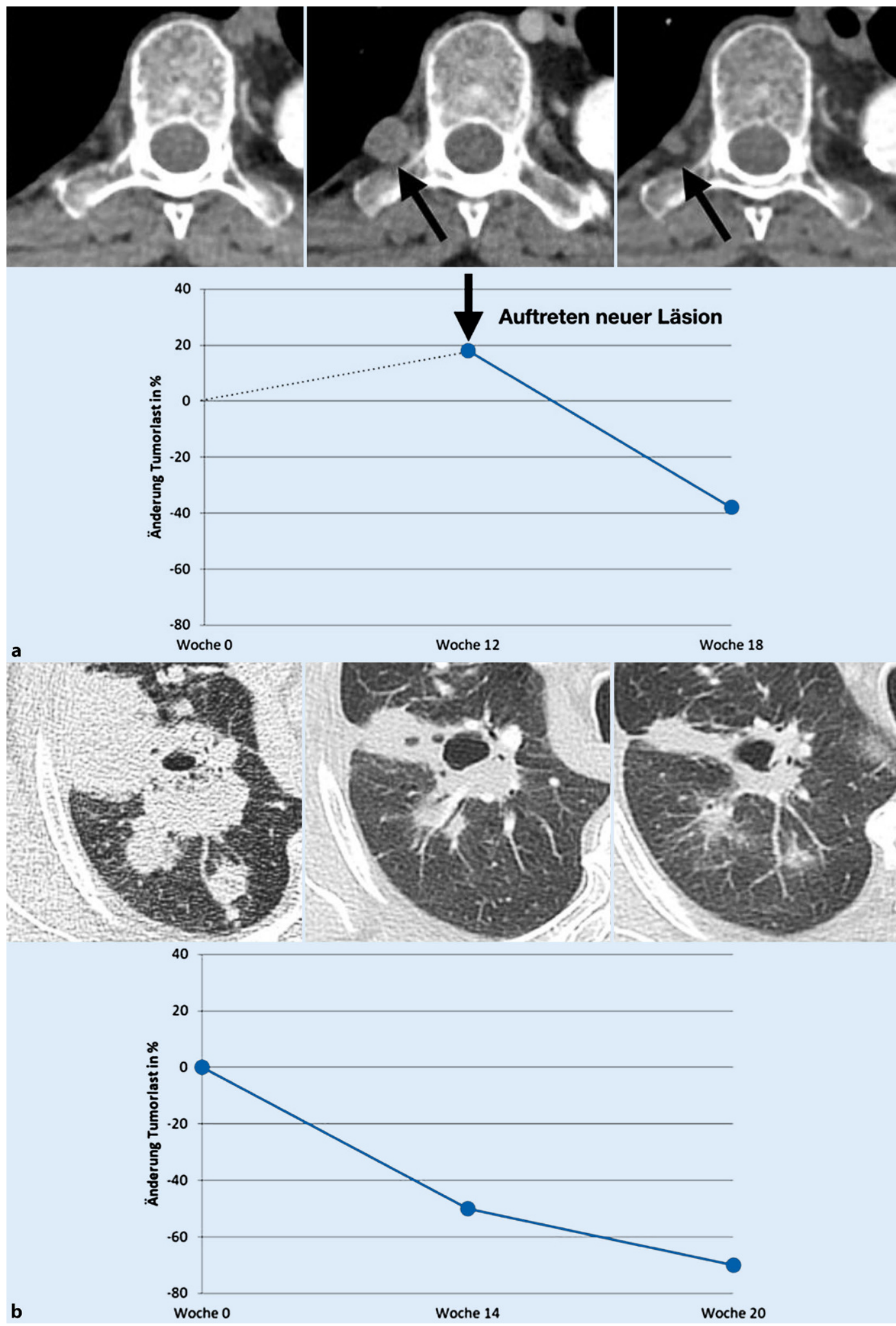

Abb. $1 \varangle$ Verschiedene Verläufe des Therapieansprechens unter Immuntherapie. a Nichtkleinzelliges Lungenkarzinom (NSCLC) unter Pembrolizumab mit Abnahme der Tumorlast $>30 \%$ in der ersten Verlaufskontrolle und folgendem weiterem Rückgang. b Pulmonale Metastase eines malignen Melanoms unter Ipilimumab mit initial geringer Zunahme der Tumorlast $<20 \%$ (SD) und nachfolgendem Regress. c NSCLC unter Pembrolizumab mit initialem Anstieg der Tumorlast $>30 \%$ und nachfolgendem Regress. d Malignes Melanom unter Nivolumab mit Auftreten einer neuen paravertebralen Metastase nach Therapieeinleitung bei ansonsten stabilem Befund. Nachfolgend Regress sowohl der neuen Läsion als auch der übrigen Tumorlast 

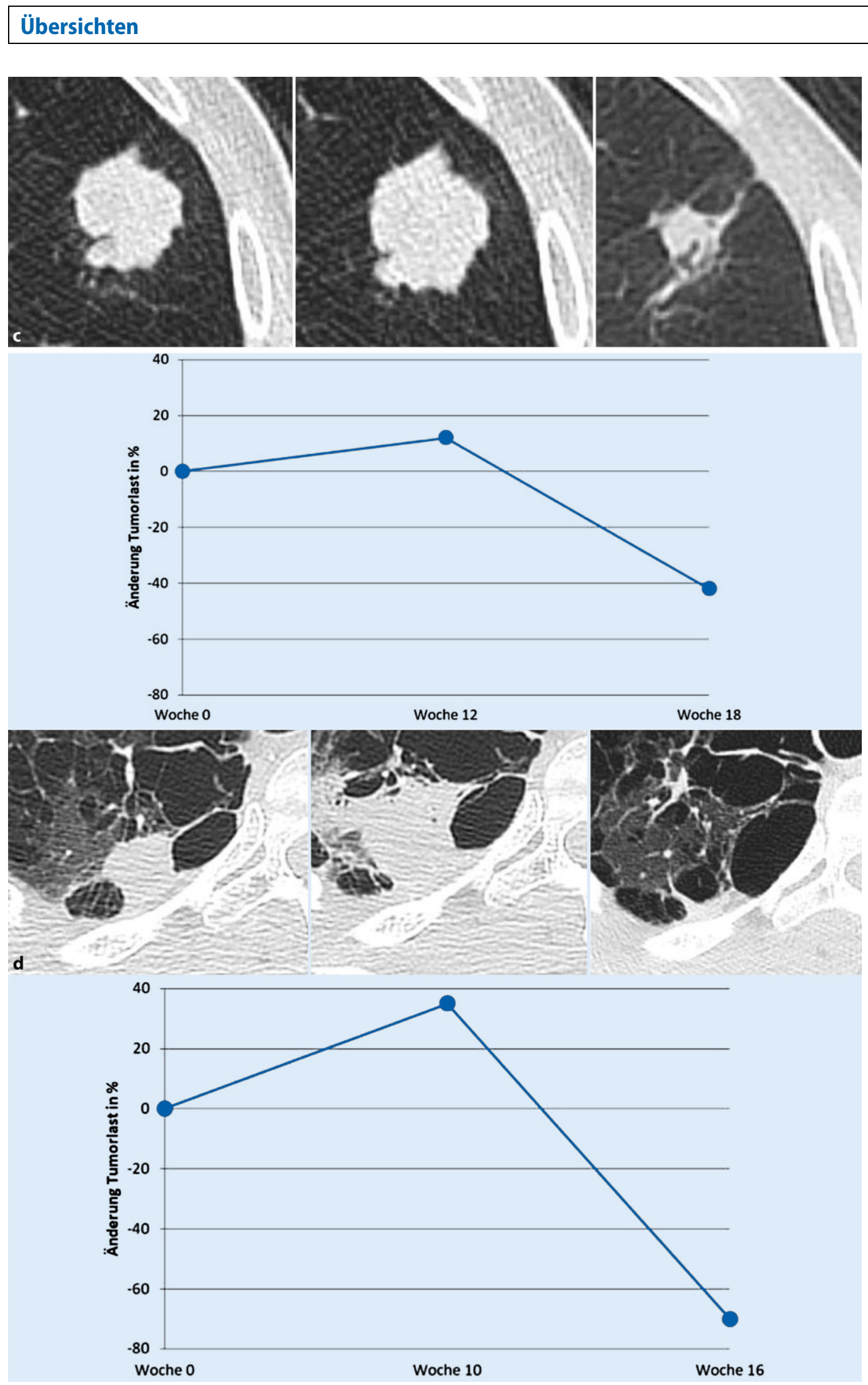

Abb. $1 \triangleleft$ (Fortsetzung) 
Tab. 2 Charakteristika verschiedener Evaluationskriterien für solide Tumoren

\begin{tabular}{|c|c|c|c|c|}
\hline & RECIST 1.1 (2009) & $\operatorname{irRC}(2009)$ & irRECIST (2013) & iRECIST (2017) \\
\hline Messung & $\begin{array}{l}\text { Unidimensional (Längs- } \\
\text { achse; bei Lymphknoten } \\
\text { Kurzachse) }\end{array}$ & $\begin{array}{l}\text { Bidimensional (analog } \\
\text { zu WHO-Kriterien) }\end{array}$ & $\begin{array}{l}\text { Unidimensional (Längsachse; } \\
\text { bei Lymphknoten Kurzachse) }\end{array}$ & $\begin{array}{l}\text { Unidimensional (Längsachse; bei Lymph- } \\
\text { knoten Kurzachse) }\end{array}$ \\
\hline $\begin{array}{l}\text { Minimale } \\
\text { Läsionsgrö- } \\
\text { Be }\end{array}$ & $\begin{array}{l}10 \text { mm Längsachse; Lymph- } \\
\text { knoten } 15 \text { mm Kurzachse }\end{array}$ & $5 \mathrm{~mm} \times 5 \mathrm{~mm}$ & $\begin{array}{l}10 \text { mm Längsachse; Lymphkno- } \\
\text { ten } 15 \text { mm Kurzachse }\end{array}$ & $\begin{array}{l}10 \text { mm Längsachse; Lymphknoten } 15 \text { mm } \\
\text { Kurzachse }\end{array}$ \\
\hline $\begin{array}{l}\text { Maximale } \\
\text { Anzahl an } \\
\text { Targetläsio- } \\
\text { nen }\end{array}$ & 5 insgesamt, 2 pro Organ & $\begin{array}{l}10 \text { insgesamt, } 5 \text { pro } \\
\text { Organ }\end{array}$ & 5 insgesamt, 2 pro Organ & 5 insgesamt, 2 pro Organ \\
\hline $\begin{array}{l}\text { Neue Läsio- } \\
\text { nen }\end{array}$ & Progress & Zur Gesamttumorlast & Zur Gesamttumorlast & Zur Gesamttumorlast \\
\hline $\begin{array}{l}\text { CR (kom- } \\
\text { plette Re- } \\
\text { mission) }\end{array}$ & $\begin{array}{l}\text { Verschwinden aller Lä- } \\
\text { sionen (LK: Kurzachse } \\
<10 \mathrm{~mm} \text { ), } \\
\text { keine neuen Läsionen }\end{array}$ & $\begin{array}{l}\text { Verschwinden aller } \\
\text { Läsionen (LK: Kurz- } \\
\text { achse }<10 \mathrm{~mm}) \text {, nach } \\
4 \text { Wochen bestätigt }\end{array}$ & $\begin{array}{l}\text { Verschwinden aller Läsionen } \\
\text { (LK: Kurzachse }<10 \mathrm{~mm} \text { ), keine } \\
\text { neuen Läsionen }\end{array}$ & $\begin{array}{l}\mathrm{iCR}=\text { Verschwinden aller Läsionen (LK: } \\
\text { Kurzachse }<10 \mathrm{~mm} \text { ), keine neuen Läsionen }\end{array}$ \\
\hline $\begin{array}{l}\text { PR (partielle } \\
\text { Remission) }\end{array}$ & $\begin{array}{l}\geq 30 \% \text { Rückgang der Tu- } \\
\text { morlast im Vergleich zur } \\
\text { Baseline }\end{array}$ & $\begin{array}{l}\geq 50 \% \text { Rückgang der } \\
\text { Tumorlast im Vergleich } \\
\text { zur Baseline; nach } \\
4 \text { Wochen bestätigt }\end{array}$ & $\begin{array}{l}\geq 30 \% \text { Rückgang der Tumorlast } \\
\text { im Vergleich zur Baseline }\end{array}$ & $\begin{array}{l}\text { iPR }=\geq 30 \% \text { Rückgang der Tumorlast im } \\
\text { Vergleich zur Baseline }\end{array}$ \\
\hline $\begin{array}{l}\text { SD (stabile } \\
\text { Erkran- } \\
\text { kung) }\end{array}$ & $\begin{array}{l}\text { Kriterien für PR oder PD } \\
\text { nicht erfüllt }\end{array}$ & $\begin{array}{l}\text { Kriterien für PR oder PD } \\
\text { nicht erfüllt }\end{array}$ & $\begin{array}{l}\text { Kriterien für PR oder PD nicht } \\
\text { erfüllt }\end{array}$ & iSD = Kriterien für PR oder PD nicht erfüllt \\
\hline $\begin{array}{l}\text { PD (Pro- } \\
\text { gress) }\end{array}$ & $\begin{array}{l}\text { Zunahme der Tumorlast } \\
\geq 20 \% \text { im Vergleich zum } \\
\text { Nadir, absolute Zunahme } \\
\geq 5 \mathrm{~mm} ; \text { Auftreten neuer } \\
\text { Läsionen }\end{array}$ & $\begin{array}{l}\geq 25 \% \text { Zunahme der } \\
\text { Tumorlast }\end{array}$ & $\begin{array}{l}\geq 20 \% \text { Zunahme der Tumorlast } \\
\text { (absolut } \geq 5 \mathrm{~mm} \text { ); Auftreten } \\
\text { neuer Läsionen; Bestätigung } \\
\text { nach } 4 \text { Wochen wird empfohlen }\end{array}$ & $\begin{array}{l}\text { iUPD }=\geq 20 \% \text { Zunahme der Tumorlast } \\
\text { (absolut } \geq 5 \mathrm{~mm} \text { ) oder Auftreten neuer } \\
\text { Läsionen in einzelner Untersuchung }\end{array}$ \\
\hline $\begin{array}{l}\text { Bestätigung } \\
\text { des Progres- } \\
\text { ses }\end{array}$ & Nicht erforderlich & $\begin{array}{l}\text { Nach } \geq 4 \text { Wochen erfor- } \\
\text { derlich }\end{array}$ & $\begin{array}{l}\text { Nach mind. } 4 \text { Wochen empfoh- } \\
\text { len }\end{array}$ & $\begin{array}{l}\text { iCPD: Bestätigung des Progresses nach } \\
4-8 \text { Wochen bei weiterer Diameterzu- } \\
\text { nahme einer Targetläsion von } \geq 5 \mathrm{~mm} \text { im } \\
\text { Vergleich zu iUPD; Auftreten weiterer neuer } \\
\text { Läsionen }\end{array}$ \\
\hline \multicolumn{5}{|c|}{ 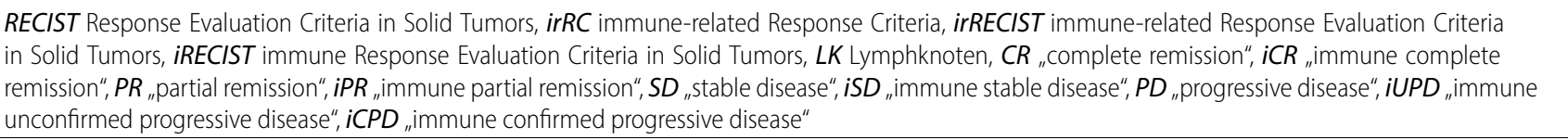 } \\
\hline
\end{tabular}

Nivolumab in Klammern), dargestellt in

- Abb. 1 [20, 39]:

1. Reduktion der Tumorlast um mehr als $30 \%$, analog zum Therapieansprechen unter klassischer zytostatischer Chemotherapie ( $80 \%)$,

2. stabile Tumorlast gefolgt von einer Reduktion (9\%),

3. Anstieg der Tumorlast um mehr als $20 \%$ gefolgt von einer Reduktion (1\%),

4. Auftreten neuer Läsionen gefolgt von einer Reduktion der Tumorlast (10\%).

Nach den aktuellen „Response Evaluation Criteria in Solid Tumors" (RECIST 1.1), welche in klinischen Studien zur quanti- tativen Evaluation eines Ansprechens der Tumorerkrankung auf die Studienmedikation genutzt werden [10], entspräche Muster 1 einer partiellen oder kompletten Remission (PR oder CR) und Muster 2 einer stabilen Erkrankung (SD). Muster 3 und 4 hingegen würden primär als Tumorprogress eingeordnet werden, weshalb hierfür der Begriff Pseudoprogress eingeführt wurde. Insgesamt sind nach obiger Definition Muster 2-4 als atypische Reaktionen zu werten. Generell bleibt anzumerken, dass unter Immuntherapie auch eine langfristig stabile Erkrankung als Therapieerfolg gewertet wird [34].

\section{Pseudoprogress}

Unter Pseudoprogress versteht man definitionsgemäß eine initiale Zunahme der Tumorlast oder ein Auftreten neuer Läsionen unter Fortführung der Therapie, gefolgt von einem Therapieansprechen im Sinne einer Reduktion der Tumorlast um mehr als $30 \%$ gegenüber der prätherapeutischen Untersuchung (Baseline). Diese Form des verzögerten Therapieansprechens ist mit den etablierten Methoden zum Therapiemonitoring (RECIST) nicht adäquat einzuordnen. Als Ursache hierfür wird zum einen angenommen, dass zunächst das weitere Tumorwachstum abgebildet wird, bevor es zu einer ausreichenden Immunreaktion und De- 


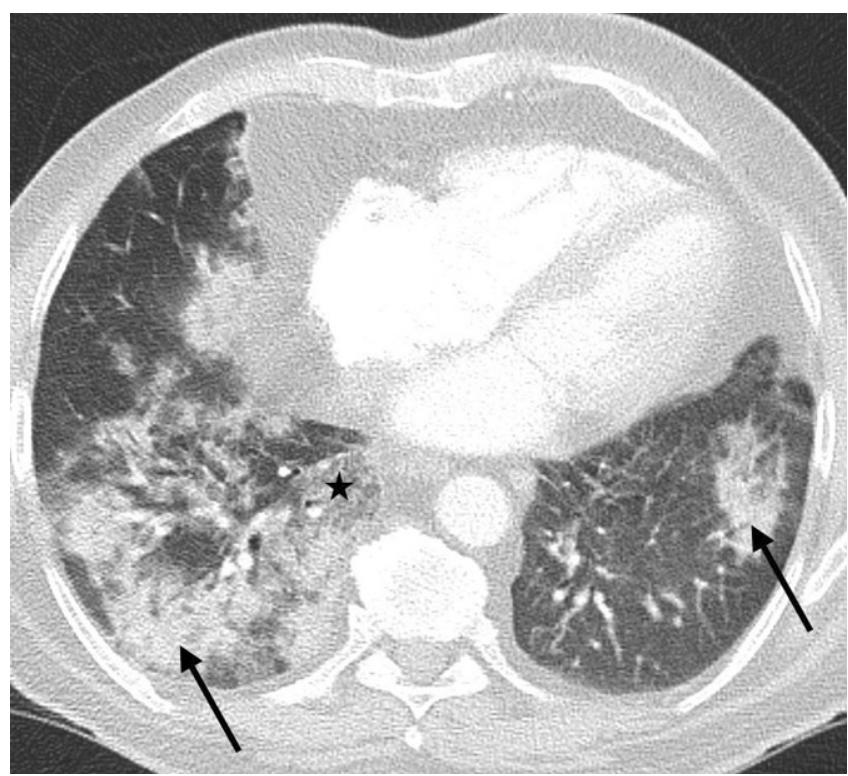

Abb. $2 \Delta$ Immuntherapieassoziierte Pneumonitis mit Muster einer kryptogenen organisierenden Pneumonie (COP): basal betonte Konsolidierungen (Pfeile) sowie hierin liegende Milchglasverdichtungen (Stern). Patient mit malignem Melanom unter Ipilimumab in der 22. Therapiewoche

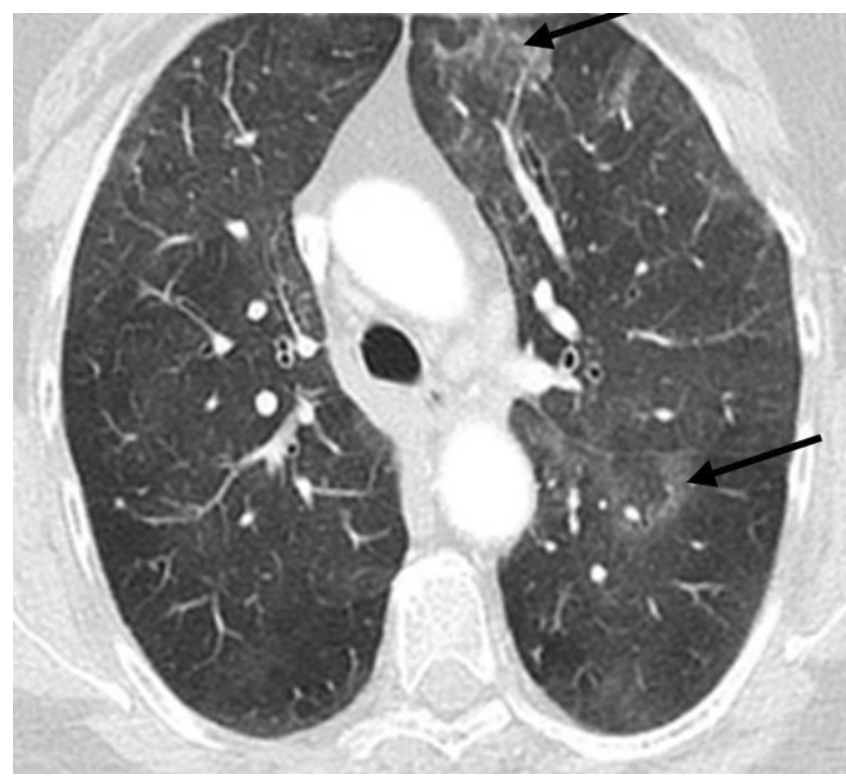

Abb. 3 A Immuntherapieassoziierte Pneumonitis mit Muster einer akuten interstitiellen Pneumonie: mosaikartig angeordnete Milchglastrübungen (Pfeile). Patientin in der 14. Woche Pembrolizumab bei Bronchialkarzinom struktion der Tumorzellen kommt. Zum anderen wird durch die Anregung des Immunsystems die T-Zell-vermittelte Akkumulation von Immunzellen im Tumor induziert, wodurch es zu einer bildmorphologischen Vergrößerung der Tumormasse oder zum Sichtbarwerden zuvor okkulter Läsionen kommen kann [10]. Ein Pseudoprogress wurde bei verschiedenen Tumorentitäten beobachtet, die Inzidenz ist insgesamt jedoch niedrig: Bei Melanomen liegt sie bei maximal $10 \%$, bei NSCLC bei $1-5 \%[9,14,25$, 45].

Nicht als Pseudoprogress, sondern nach aktuellem Konsens noch als stabile Erkrankung gilt eine Zunahme der Tumorlast unter Immuntherapie um weniger als $20 \%$.

Ebenfalls nicht als Pseudoprogress wird gewertet, wenn die Tumorlast nach initialem Anstieg zwar zurückgeht, die für den Pseudoprogress geforderten $30 \%$-Rückgang jedoch nicht erreicht und somit keine Unterschreitung der Baseline Tumorlast erzielt wird. Für die prognostische Bewertung der letztgenannten Gruppe, die formell als progredient gewertet werden müsste, fehlen aktuell suffiziente Daten; dagegen zeigen Patienten mit einem initial nur geringen Anstieg der Tumorlast im Sinne einer noch stabilen Erkrankung $(<20 \%)$ ein tendenziell verbessertes Gesamtüberleben [25, 32].

Der initiale Anstieg der Tumorlast unter Immuntherapie wird typischerweise zwischen 3 Monaten und 1 Jahr nach Therapieeinleitung beobachtet, die darauffolgende Remission zumeist innerhalb von 3 Monaten. In wenigen Fällen war unter Fortführung der Therapie jedoch auch ein deutlich späterer Rückgang der Tumorlast bis zu mehreren Monaten nach deren initialem Anstieg möglich [14,27]. Insgesamt erlaubt die aktuelle Datenlage keine einheitliche Festlegung eines Zeitraums, in welchem ein Pseudoprogress auftreten kann. Zusätzlich fehlt derzeit noch die Evidenz, welche Zeitpunkte für Folgeuntersuchungen zur weiteren Differenzierung zu empfehlen sind. Der initiale Vorschlag eines Intervalls von mindestens 4 Wochen durch Wolchok wurde in der nachfolgenden Literatur als Konsensempfehlung zunächst weitgehend übernommen, in neueren Publikation wird eine Verlängerung des Intervalls diskutiert $[26,32,46]$. In jedem Fall ist eine interdisziplinäre, individuelle Entscheidung über Therapiefortsetzung oder -abbruch unter Einbezug des klinischen Verlaufs essenziell [31, 46].
Auch nuklearmedizinische Techniken können bisher nicht sicher zwischen Progress und Pseudoprogress differenzieren: Zwar konnte in einigen Fällen eine erhöhte Traceraufnahme im FDGPET-CT bei einem echten Progress gezeigt werden, die nur geringe Spezifität einer Stoffwechselsteigerung für einen tatsächlichen Tumorprogress limitiert allerdings einen Nutzen der PET in der klinischen Routine [2, 22]. Die Erforschung neuer Tracer, welche die Immunaktivität oder Zellproliferationen beispielsweise über radioaktiv markierte Anti-CD-8-Antikörper oder markiertes Fluorothymidin (FLT-PET) darstellen, könnte zukünftig die Möglichkeit einer nichtinvasiven einzeitigen Differenzierung eines echten Progresses von einem Pseudoprogress ermöglichen [1, 38]. Auch könnten zukünftig RadiomicsAnalysen zur Identifikation bildgebender Biomarker zu einer besseren Differenzierung des Therapieansprechens beitragen [4].

\section{Hyperprogress}

Neben atypischen Verläufen bei Therapieansprechen wurde auch bei echtem Tumorprogress ein neues Phänomen beobachtet: eine übermäßige Zunahme der 


\begin{tabular}{l|l|l}
$\begin{array}{l}\text { Tab. } 3 \\
\text { Events) }\end{array}$ & Gradeinteilung der Pneumonitis nach CTCAE (Common Terminology Criteria for Adverse \\
\hline Grad & Klinik & CT Thorax \\
\hline 1 & Asymptomatisch & $\begin{array}{l}\text { Beschränkt auf 1 Lungenlappen oder } \\
<25 \% \text { des Parenchyms }\end{array}$ \\
2 & $\begin{array}{l}\text { Milde Symptome, medizinische Behandlung } \\
\text { notwendig }\end{array}$ & $\begin{array}{l}\text { Min. } 2 \text { Lungenlappen oder } \\
25-50 \% \text { des Parenchyms involviert }\end{array}$ \\
3 & $\begin{array}{l}\text { Schwere Symptome, stationäre Behandlung } \\
\text { notwendig, Sauerstoffgabe }\end{array}$ & $\begin{array}{l}\text { Alle Lungenlappen oder } \\
>50 \% \text { des Parenchyms involviert }\end{array}$ \\
4 & $\begin{array}{l}\text { Lebensbedrohliche Symptome, Intubations- } \\
\text { pflicht }\end{array}$ & $\begin{array}{l}\text { Alle Lungenlappen oder } \\
>50 \% \text { des Parenchyms involviert } \\
\text { Alle Lungenlappen oder }\end{array}$ \\
\hline 5 & Tod & $>50 \%$ des Parenchyms involviert
\end{tabular}

Tumorzelllast unter Immuntherapie, welche unter konventioneller Chemotherapie nicht erwartet würde. Für diesen sogenannten Hyperprogress existiert bisher keine einheitliche Definition - je nach Autor wird eine doppelte Wachstumsrate im Vergleich zur Wachstumsrate vor Therapieeinleitung oder ein $>50 \%$ iger Anstieg der Diameter in 2 verschiedenen Läsionen innerhalb von 2 Monaten als solcher definiert $[8,17]$. Die Inzidenzen bewegen sich je nach Definition und Tumorentität im Bereich von 6-14\%, bei Plattenepithelkarzinomen des Kopf- und Halsbereichs sogar bei $29 \%[6,8,17]$. Es konnte eine Assoziation mit einem erhöhten Lebensalter ( $>65$. Lebensjahr) gezeigt werden, außerdem prädisponieren eine vorangegangene Radiotherapie, multiple Metastasenlokalisationen oder bestimmte Genmutationen für einen $\mathrm{Hy}$ perprogress $[8,13,17]$. Unklar ist, ob die deutliche onkologische Verschlechterung durch die Immuntherapie induziert bzw. verstärkt wird, oder ob sie lediglich den natürlichen Verlauf unter ineffizienter Therapie darstellt. Da es an einer strukturierten Aufarbeitung des Phänomens derzeit mangelt, steht eine Beantwortung dieser Frage aus.

\section{Neue Evaluationskriterien des Therapieansprechens}

Aufgrund der beschriebenen Therapieverläufe wurde schon in den ersten klinischen Studien vermutet, dass eine Evaluation des Therapieansprechens mittels klassischer Kriterien problembehaftet sein würde. Retrospektive Analysen ergaben bei $15 \%$ der Patienten, welche in der der ersten Bildgebung nach Therapieeinleitung als Progress nach RECIST 1.1 gewertet wurden, unter Fortführung der Therapie einen nachfolgenden Rückgang [15]. Als erster Versuch einer Lösung wurden daher 2009 „immune-related Response Criteria“ (irRC) vorgeschlagen, die eine Bestätigung des Progresses nach mindestens 4 Wochen fordern. Bei Auftreten neuer Läsionen führt dies jedoch nicht automatisch zur Feststellung eines Progresses, sondern dazu, dass deren Maße lediglich der Gesamttumorlast zugeschlagen werden [46]. Da die Kriterien jedoch auf einer bidimensionalen Messung, analog zu den WHO-Kriterien, beruhen und somit die Vergleichbarkeit gegenüber RECIST 1.1 deutlich eingeschränkt ist, wurden bald die „immunerelated RECIST“ (irRECIST) entwickelt [26], welche die gleichen Neuerungen enthalten, jedoch basierend auf eindimensionaler Messung der Längsachse bei Tumorknoten und Kurzachse bei Lymphknoten, analog zu RECIST 1.1. Im Jahr 2017 wurden durch die RECISTArbeitsgruppe zusätzlich die „immune RECIST“ (iRECIST) veröffentlicht [36]. Diese enthalten neben den Änderungen wie in irRECIST neue Kategorien zur Beurteilung des Therapieansprechens, welche in $\bullet$ Tab. 2 genauer erläutert werden. Wesentliche Neuerung ist die Unterteilung der Kategorie „Tumorprogress (PD)“ in einen unbestätigten (iUPD, U: „unconfirmed“) und einen bestätigten Progress (iCPD) (C: „confirmed“). Dabei ist ein Zeitintervall von 4-8 Wochen zur Bestätigung des Progresses vorgesehen, für den eine weitere Größenzunahme einer Läsion um min- destens 5 mm oder das Auftreten weiterer neuer Läsionen gefordert ist. Ergibt sich in der Folgeuntersuchung ein unveränderter Befund, so bleibt die Kategorie iUPD bis zu dessen Bestätigung bestehen [6].

Obwohl für irRECIST im Vergleich zu den iRC zu einem verlängerten Intervall bis zu einer Progress-bestätigenden Untersuchung geraten wird, können selbst 8 Wochen in Einzelfällen nicht sicherstellen, dass ein verzögertes Therapieansprechen adäquat gewürdigt wird.

Einen Überblick über die verwendeten Evaluationskriterien gibt • Tab. 2.

\section{Monitoring immuntherapie- induzierter Nebenwirkungen}

Aufgrund des Wirkmechanismus unterscheiden sich auch die unter Immuntherapie auftretenden Nebenwirkungen von denjenigen der klassischen zytostatischen Chemotherapie. Schwere bis lebensbedrohliche Nebenwirkungen (Grad 3-4), welche einen Therapieabbruch nötig machen, treten dabei in 9-15\% der Fälle auf und sind für CTLA4-Inhibitoren häufiger als für PD-1- oder PD-L1-Inhibitoren [7, 11, 16, 40]. Da es sich bei vielen der Nebenwirkungen um entzündliche Reaktionen handelt, liegt es nahe, dass es sich um Autoimmunphänomene handelt [28]. Die häufigsten Manifestationen sind Diarrhöen, Dermatitis und andere Hautreaktionen, Hypophysitis, Hypothyreose, Pneumonitis, Hepatitis und Lymphadenopathie, seltener treten Entzündungen von ZNS, Augen, Gelenken, Nieren, Pankreas oder des Myokards auf [5, 28]. Von Bedeutung ist die Bildgebung vor allem in der Diagnostik der Pneumonitis und der Hypophysitis, weshalb auf diese noch genauer eingegangen wird. Darüber hinaus ist die therapiebegleitende Bildgebung hilfreich bei der Differenzierung von Nebenwirkung und Komplikation der Grunderkrankung.

Die mit ca. 20 \% häufigste Nebenwirkung einer Diarrhö ist zwar eine rein klinische Diagnose, bei abdominellen Beschwerden kann jedoch eine CT-Untersuchung des Abdomens indiziert sein [5, 7]. Im Fall einer Kolitis stellt sich diese in der Regel als diffuse, ödematöse 
Tab. 4 Erscheinungsformen und relative Häufigkeiten der immuntherapieassoziierten Pneumonitis

\begin{tabular}{|c|c|c|c|}
\hline Muster & Interstitielle Veränderung & Verteilung & $\begin{array}{l}\text { Relative Häu- } \\
\text { figkeit }\end{array}$ \\
\hline $\begin{array}{l}\text { Unspezifische interstitielle Pneumonie } \\
\text { (NSIP) }\end{array}$ & $\begin{array}{l}\text { Milchglasverdichtungen, retikuläre Verdichtungen, Bron- } \\
\text { chiektasien }\end{array}$ & $\begin{array}{l}\text { Basal, subpleurale Aus- } \\
\text { sparungen }\end{array}$ & $8-22 \%$ \\
\hline $\begin{array}{l}\text { Akute interstitielle Pneumonie (AIP)/ } \\
\text { diffuser Alveolarschaden (ARDS) }\end{array}$ & Milchglasverdichtungen, Konsolidierungen & Mosaikartig & $10 \%$ \\
\hline Hypersentitivitätspneumonie (HP) & $\begin{array}{l}\text { Retikuläre Konsolidierungen mit oder ohne Milchglasver- } \\
\text { dichtungen, noduläre Verdichtungen möglich }\end{array}$ & Oberlappen & $7-16 \%$ \\
\hline $\begin{array}{l}\text { Gewöhnliche interstitielle Pneumonie } \\
\text { (UIP) }\end{array}$ & Diffuse retikuläre Verdichtungen & Lungenperipherien & Selten \\
\hline $\begin{array}{l}\text { Kryptogene organisierende Pneumonie } \\
\text { (COP) }\end{array}$ & $\begin{array}{l}\text { Alveoläre Konsolidierungen, inverses Halo-Zeichen mög- } \\
\text { lich }\end{array}$ & $\begin{array}{l}\text { Peribronchovaskulär, } \\
\text { subpleural, basal }\end{array}$ & $19-65 \%$ \\
\hline
\end{tabular}

Schwellung der Darmwand mit Kontrastmittelaufnahme und begleitender Umgebungsreaktion dar. Zeigt sich das Bild einer klassischen Divertikulitis, so ist auch hier die Differenzialdiagnose einer immuntherapieassoziierten Nebenwirkung $\mathrm{zu}$ nennen, eine Unterscheidung ist allein anhand der Schnittbildgebung nicht möglich $[19,44]$. Auch eine Lymphadenopathie mit meist hilärer und/oder mediastinaler Manifestation, auch als Sarkoidose-ähnliche Reaktion bezeichnet, ist mit einer Häufigkeit von etwa $5 \%$ eine häufige Nebenwirkung von Immuntherapien [28]. In der radiologischen Diagnostik sollte diese als Differenzialdiagnose eines möglichen malignen Befalls oder einer chronisch-entzündlichen Lymphknotenvergrößerung anderer Ursache in Erwägung gezogen werden.

\section{Pneumonitis}

Die Pneumonitis ist als Nebenwirkung von Immuntherapien mit Inzidenzen bis 3\% insgesamt eher selten, aufgrund des oft schwerwiegenden und mitunter letalen Verlaufs kommt ihr jedoch eine besondere Bedeutung zu [5, 18]. Gehäuft tritt sie bei respiratorisch vorerkrankten Patienten (inklusive NSCLC), bei Patienten nach thorakaler Bestrahlung sowie bei Behandlung mit PD-1-Inhibitoren oder einer Kombinationstherapie mehrerer Immuntherapeutika auf $[18,30]$, zumeist 2 Wochen bis 12 Monate (Median: 2,6 Monate) nach Therapieeinleitung [30]. Klinisch stehen ein trockener Husten, Dyspnoe und eine Verschlechterung der respiratorischen Funktion im Vor- dergrund. Radiologisch kann sich eine Pneumonitis in den unterschiedlichsten Formen präsentieren, wobei die computertomographischen Erscheinungsbilder den Mustern der verschiedenen interstitiellen Pneumonien entsprechen. Gemeinsames Merkmal in den meisten Fällen sind dabei milchglasartige Dichteanhebungen des Lungenparenchyms [29]. Die weiteren häufigsten Veränderungen entsprechen denen einer organisierenden Pneumonie (COP) oder einer unspezifischen interstitiellen Pneumonie (NSIP; [3, 24]). In - Abb. 2 und 3 sind typische Erscheinungsformen dargestellt. Einen Überblick über die Schweregrade und die Erscheinungsformen der immuntherapieinduzierten Pneumonitis geben $\bullet$ Tab. 2, 3 und 4 [24, 41-43].

Außerdem kann bei thorakal bestrahlten Patienten durch Immuntherapien eine Verschlimmerung oder ein Wiederauftreten abgeklungener radiogener Pneumonitiden induziert werden, welche sich als rasche Zunahme bzw. ein Wiederauftreten typischer radiogener Veränderungen im Bestrahlungsfeld mit oder ohne begleitende Zeichen der immuntherapieinduzierten Pneumonitis außerhalb des Bestrahlungsfelds darstellen [35]. Vergleichbare Reaktionen die neuerliche Aggravation radiogener Veränderungen im Rahmen einer Immuntherapie - kann auch in anderen Körperregionen beobachtet werden.

Ein weiteres Phänomen ist das sog. „pneumonitis flare“, mit welchem ein erneutes Auftreten von pneumonitischen Symptomen und bildmorphologischen
Veränderungen nach Beendigung einer Immuntherapie bei vorangegangener, steroidal behandelter Pneumonitis beschrieben wird.

Die Kenntnis dieser Erscheinungsformen ist von radiologischer Seite insbesondere bei thorakalen Tumoren notwendig, da die Differenzierung pneumonitischer Veränderungen von einer progredienten oder neuen Tumormanifestation oft eine diagnostische Herausforderung darstellt und einer gründlichen Befundung und Expertise bedarf. Darüber hinaus stellt eine infektiöse Ursache der Lungenparenchymveränderungen eine häufige Differenzialdiagnose dar, welche meist nur im interdisziplinären Zusammenhang ausgeschlossen werden kann.

\section{Hypophysitis}

Die Hypophysitis trittbei 10-13\% der mit Ipilimumab behandelten Patienten auf und stellt damit eine häufige Nebenwirkung dar; unter PD-1- bzw. PD-L1-Inhibitoren ist sie mit $<1 \%$ hingegen eher selten $[5,12]$. Klinisch präsentiert sie sich als neu aufgetretene Hypophysenvorderlappeninsuffizienz meist mit Kopfschmerzen und/oder Fatigue 1 bis 9 Monate (Median: 9 Wochen) nach Therapieeinleitung. Zeigt sich zusätzlich in der MRT das Bild einer vergrößerten Hypophyse, ggf. mit homo- oder heterogener Kontrastmittelaufnahme und verdicktem Infundibulum, so kann von einer immuntherapieinduzierten Hypophysitis ausgegangen werden. Eine neu aufgetretene Hypophysenvergrößerung kann auch ohne 


\begin{tabular}{|c|c|}
\hline \multicolumn{2}{|c|}{ Abkürzungen } \\
\hline$C O P$ & $\begin{array}{l}\text { "Cryptogenic organizing pneumo- } \\
\text { nia" }\end{array}$ \\
\hline$C R$ & $\begin{array}{l}\text { "Complete remission“ (vollständi- } \\
\text { ges Therapieansprechen) }\end{array}$ \\
\hline$C T$ & Computertomographie \\
\hline CTLA-4 & $\begin{array}{l}\text { "Cytotoxic T-lymphocyte-associa- } \\
\text { ted protein-4" }\end{array}$ \\
\hline$F D G$ & Fluordesoxyglycose \\
\hline FLT & Fluorthymidin \\
\hline$i C P D$ & $\begin{array}{l}\text { „Immune confirmed progressive } \\
\text { disease“ (bestätigter Krankheits- } \\
\text { progress) }\end{array}$ \\
\hline iRECIST & $\begin{array}{l}\text { "Immune response evaluation } \\
\text { criteria in solid tumors" }\end{array}$ \\
\hline irRC & $\begin{array}{l}\text { "Immune-related response } \\
\text { criteria" }\end{array}$ \\
\hline irRECIST & $\begin{array}{l}\text { „Immune-related response } \\
\text { evaluation criteria in solid tumors" }\end{array}$ \\
\hline iUPD & $\begin{array}{l}\text { "Immune unconfirmed pro- } \\
\text { gressive disease“ (unbestätigter } \\
\text { Krankheitsprogress) }\end{array}$ \\
\hline MRT & Magnetresonanztomographie \\
\hline$N S C L C$ & $\begin{array}{l}\text { "Non small cell lung cancer" } \\
\text { (nichtkleinzelliges Lungenkarzi- } \\
\text { nom) }\end{array}$ \\
\hline NSIP & $\begin{array}{l}\text { „Nonspecific interstitial Pneumo- } \\
\text { nia“ }\end{array}$ \\
\hline$P D$ & $\begin{array}{l}\text { „Progressive disease“ (Krankheits- } \\
\text { progress) }\end{array}$ \\
\hline$P D-1$ & $\begin{array}{l}\text { "Programmed cell death prote- } \\
\text { in-1" }\end{array}$ \\
\hline$P D-L 1$ & $\begin{array}{l}\text { "Programmed cell death protein-1 } \\
\text { ligand-1" }\end{array}$ \\
\hline PET & Positronenemmisionstomographie \\
\hline$P R$ & $\begin{array}{l}\text { „Partial response" (teilweises } \\
\text { Therapieansprechen) }\end{array}$ \\
\hline RECIST & $\begin{array}{l}\text { "Response evaluation criteria in } \\
\text { solid tumors" }\end{array}$ \\
\hline$S D$ & $\begin{array}{l}\text { "Stable disease" (stabile Erkran- } \\
\text { kung) }\end{array}$ \\
\hline ZNS & Zentrales Nervensystem \\
\hline
\end{tabular}

zuvor aufgefallene hormonelle Störung auf eine Hypophysitis hindeuten. Therapeutisch reichen Kortison und ein Hormonersatz zumeist aus, um eine Remission zu erzielen, wobei die Hypophyseninsuffizienz bei drei Viertel der Patienten persistiert [12].

\section{Fazit für die Praxis}

- Eine adäquate Verlaufsbeurteilung ist nur unter Berücksichtigung des Immuntherapeutikums, der Therapiedauer sowie etwaiger Vortherapien im Vergleich mit allen vorliegenden Voruntersuchungen möglich.

- Die Gesamttumorlast und ihre Änderung werden durch Messung einzelner Läsionen unter Berücksichtigung des Gesamtbilds eingeschätzt.

- Je nach Beurteilung ergeben sich daraus folgende Konsequenzen: a) Zunahme der Gesamttumorlast um $\geq 100 \%$ bzw. mehr als ohne Therapie erwartet: V.a. Hyperprogress.

b) Deutliche Zunahme der Gesamttumorlast (um $\geq 20 \%$ ) und Zunahme einer Läsion um $\geq 5 \mathrm{~mm}$ oder Auftreten neuer Läsionen: Folgeuntersuchung in 6-8 Wochen zur Bestätigung eines Progresses. Gegebenenfalls sind weitere Verlaufskontrollen notwendig. c) Weitgehend unveränderter Befund oder Rückgang der Tumorlast: reguläre Verlaufskontrolle nach 12 Wochen.

- Beachte: Mit einer Inzidenz von $<10 \%$ bleibt der Pseudoprogress selten.

- Auf typische Veränderungen im Rahmen immuntherapieassoziierter Nebenwirkungen ist zu achten.

\section{Korrespondenzadresse}

\section{Michel Eisenblätter}

Klinik für Diagnostische und Interventionelle Radiologie, Universitätsklinikum Freiburg Hugstetter Str. 55, 79106 Freiburg, Deutschland michel.eisenblaetter@uniklinik-freiburg.de

Funding. Open Access funding provided by Projekt DEAL.

\section{Einhaltung ethischer Richtlinien}

Interessenkonflikt. H. Schneider, A.-L. Illert, F. Bamberg und M. Eisenblätter geben an, dass kein Interessenkonflikt besteht.

Für diesen Beitrag wurden von den Autoren keine Studien an Menschen oder Tieren durchgeführt. Für die aufgeführten Studien gelten die jeweils dort angegebenen ethischen Richtlinien.
Open Access. Dieser Artikel wird unter der Creative Commons Namensnennung 4.0 International Lizenz veröffentlicht, welche die Nutzung, Vervielfältigung Bearbeitung, Verbreitung und Wiedergabe in jeglichem Medium und Format erlaubt, sofern Sie den/die ursprünglichen Autor(en) und die Quelle ordnungsgemäß nennen, einen Link zur Creative Commons Lizenz beifügen und angeben, ob Änderungen vorgenommen wurden.

Die in diesem Artikel enthaltenen Bilder und sonstige Drittmaterial unterliegen ebenfalls der genannten Creative Commons Lizenz, sofern sich aus der Abbildungslegende nichts anderes ergibt. Sofern das betreffende Material nicht unter der genannten Creative Commons Lizenz steht und die betreffende Handlung nicht nach gesetzlichen Vorschriften erlaubt ist, ist für die oben aufgeführten Weiterverwendungen des Materials die Einwilligung des jeweiligen Rechteinhabers einzuholen.

Weitere Details zur Lizenz entnehmen Sie bitte der Lizenzinformation auf http://creativecommons.org/ licenses/by/4.0/deed.de.

\section{Literatur}

1. Aarntzen EHJG, Srinivas M, Radu CG et al (2013) In vivo imaging of therapy-induced anti-cancer immune responses in humans. Cell Mol Life Sci 70:2237-2257. https://doi.org/10.1007/s00018012-1159-2

2. Aide N, Hicks RJ, Le Tourneau C et al (2019) FDG PET/CT for assessing tumour response to immunotherapy: Report on the EANM symposium on immune modulation and recent review of the literature. Eur J Nucl Med Mol Imaging 46:238-250. https://doi.org/10.1007/s00259-018-4171-4

3. Akella P, Loganathan S, Jindal V et al (2019) Anti PD-1 immunotherapy related interstitial lung disease presenting as respiratory failure-A review with case series. Respir Med Case Rep 26:17-22. https://doi.org/10.1016/j.rmcr.2018.11 005

4. Banna GL, Olivier T, Rundo F et al (2019) The promise of digital biopsy for the prediction of tumor molecular features and clinical outcomes associated with immunotherapy. Front Med 6:172 https://doi.org/10.3389/fmed.2019.00172

5. Baxi S, Yang A, Gennarelli RL et al (2018) Immunerelated adverse events for anti-PD-1 and anti-PDL1 drugs: systematic review and meta-analysis. BMJ.https://doi.org/10.1136/bmj.k793

6. Borcoman E, Nandikolla A, Long G et al (2018) Patterns of response and progression to immunotherapy. Am Soc Clin Oncol Educ Book. https://doi. org/10.1200/EDBK_200643

7. Brahmer JR, TykodiSS, ChowLQMetal (2012) Safety and activity of anti-PD-L1 antibody in patients with advanced cancer. N Engl J Med 366:2455-2465. https://doi.org/10.1056/NEJMoa1200694

8. Champiat S, Ferrara R, Massard C et al (2018) Hyperprogressive disease: recognizing a novel pattern to improve patient management. Nat Rev Clin Oncol 15:748-762. https://doi.org/10.1038/ s41571-018-0111-2

9. Chiou VL, Burotto M (2015) Pseudoprogression and immune-related response in solid tumors. J Clin Oncol 33:3541-3543. https://doi.org/10. 1200/JCO.2015.61.6870

10. Eisenhauer EA, Therasse P, Bogaerts J et al (2009) New response evaluation criteria in solid tumours: 
Revised RECIST quideline (version 1.1). Eur J Cancer 45:228-247. https://doi.org/10.1016/j.ejca.2008. 10.026

11. El Osta B, Hu F, Sadek R et al (2017) Not all immune-checkpoint inhibitors are created equal: Meta-analysis and systematic review of immunerelated adverse events in cancer trials. Crit Rev Oncol Hematol 119:1-12.https://doi.org/10.1016/ j.critrevonc.2017.09.002

12. Faje AT, Sullivan R, Lawrence D et al (2014) Ipilimumab-induced Hypophysitis: a detailed longitudinal analysis in a large cohort of patients with metastatic melanoma. J Clin Endocrinol Metab 99:4078-4085. https://doi.org/10.1210/jc. 2014-2306

13. Ferrara R, Mezquita L, Texier M et al (2018) Hyperprogressive disease in patients with advanced non-small cell lung cancer treated with PD-1/PDL1 inhibitors or with single-agent chemotherapy. JAMA Oncol 4:1543. https://doi.org/10.1001/ jamaoncol.2018.3676

14. Hodi FS, Hwu W-J, Kefford Ret al (2016) Evaluation of immune-related response criteria and RECIST v1.1 in patients with advanced melanoma treated with Pembrolizumab. J Clin Oncol 34:1510-1517. https://doi.org/10.1200/JCO.2015.64.0391

15. Hodi FS, Oble DA, Drappatz J et al (2008) CTLA-4 blockade with ipilimumab induces significant clinical benefit in a female with melanoma metastases to the CNS. Nat Clin Pract Oncol 5:557-561. https://doi.org/10.1038/ncponc1183

16. Hodi FS, O'Day SJ, McDermott DF et al (2010) Improved survival with Ipilimumab in patients with metastatic melanoma. N Engl J Med 363:711-723. https://doi.org/10.1056/NEJMoa1003466

17. Kato S, Goodman A, Walavalkar V et al (2017) Hyperprogressors after immunotherapy: analysis of genomic alterations associated with accelerated growth rate. Clin Cancer Res 23:4242-4250. https://doi.org/10.1158/1078-0432.CCR-16-3133

18. Khunger M, Pasupuleti V, Rakshit S et al (2017) Incidence of pneumonitis with use of PD-1 and PD-L1 inhibitors in non-small cell lung cancer: A systematic review and meta-analysis of trials. J Clin Oncol 35:e20647-e20647. https://doi.org/ 10.1200/JCO.2017.35.15_suppl.e20647

19. Kim Y-Y, Kim M-J, Kim EH etal (2019) Hepatocellular carcinoma versus other hepatic malignancy in cirrhosis: performance of LI-RADS version 2018 Radiology 291:72-80. https://doi.org/10.1148/ radiol.2019181995

20. Kwak JJ, Tirumani SH, Van den Abbeele AD et al (2015) Cancer immunotherapy: imaging assessment of novel treatment response patterns and immune-related adverse events. Radiographics 35:424-437. https://doi.org/10.1148/rg. 352140121

21. Kwok G, Yau TCC, Chiu JW et al (2016) Pembrolizumab (Keytruda). Hum Vaccines Immunother 12:2777-2789. https://doi.org/10.1080/ 21645515.2016.1199310

22. Ma W, Ji Y, Qi L et al (2018) Breast cancer Ki67 expression prediction by DCE-MRI radiomics features. Clin Radiol 73:909.e1-909.e5. https://doi. org/10.1016/j.crad.2018.05.027

23. Mok TSK, Wu Y-L, Kudaba I et al (2019) Pembrolizumab versus chemotherapy for previously untreated, PD-L1-expressing, locally advanced or metastatic non-small-cell lung cancer (KEYNOTE042): a randomised, open-label, controlled, phase 3 trial. Lancet 393:1819-1830. https://doi.org/10. 1016/S0140-6736(18)32409-7

24. Naidoo J, Wang X, Woo KMetal (2017) Pneumonitis in patients treated with anti-programmed death- 1/programmed death Ligand 1 therapy. J Clin Oncol 35:709-717. https://doi.org/10.1200/JCO. 2016.68.2005

25. Nishino M, Dahlberg SE, Adeni AE et al (2017) Tumor response dynamics of advanced nonsmall cell lung cancer patients treated with PD-1 inhibitors: imaging markers for treatment outcome. Clin Cancer Res 23:5737-5744. https:// doi.org/10.1158/1078-0432.CCR-17-1434

26. Nishino M, Giobbie-Hurder A, Gargano $M$ et al (2013) Developing a common language for tumor response to immunotherapy: immunerelated response criteria using Unidimensional measurements. Clin Cancer Res 19:3936-3943. https://doi.org/10.1158/1078-0432.CCR-13-0895

27. Nishino M, Giobbie-Hurder A, Manos MP et al (2017) Immune-related tumor response dynamics in melanoma patients treated with Pembrolizumab: identifying markers for clinical outcome and treatment decisions. Clin Cancer Res 23:4671-4679. https://doi.org/10.1158/1078 0432.CCR-17-0114

28. Nishino $M$, Hatabu $H$, Hodi FS (2019) Imaging of cancer immunotherapy: current approaches and future directions. Radiology 290:9-22. https://doi. org/10.1148/radiol.2018181349

29. Nishino M, Hatabu H, Sholl LM, Ramaiya NH (2017) Thoracic complications of precision cancer therapies: a practical guide for radiologists in the new era of cancer care. Radiographics 37:1371-1387. https://doi.org/10.1148/rg.2017170015

30. Nishino M, Ramaiya NH, Awad MMetal (2016) PDinhibitor-related pneumonitis in advanced cancer patients: radiographic patterns and clinical course. Clin Cancer Res 22:6051-6060. https://doi.org/10. 1158/1078-0432.CCR-16-1320

31. Nishino M, Ramaiya NH, Hatabu H, Hodi FS (2017) Monitoring immune-checkpoint blockade: response evaluation and biomarker development. NatRev Clin Oncol 14:655-668. https://doi.org/10 1038/nrclinonc.2017.88

32. Okada H, Weller M, Huang R et al (2015) Immunotherapy response assessment in neurooncology: a report of the RANO working group. Lancet Oncol 16:e534-e542. https://doi.org/10. 1016/S1470-2045(15)00088-1

33. Ott PA, Hodi FS, Robert C (2013) CTLA-4 and PD-1/PD-L1 blockade: new Immunotherapeutic modalities with durable clinical benefit in melanomapatients.Clin CancerRes 19:5300-5309. https://doi.org/10.1158/1078-0432.CCR-13-0143

34. Pardoll DM (2012) The blockade of immune checkpoints in cancer immunotherapy. Nat Rev Cancer 12:252-264. https://doi.org/10.1038/ nrc3239

35. Schoenfeld JD, Nishino M, Severgnini Met al (2019) Pneumonitis resulting from radiation and immune checkpoint blockade illustrates characteristic clinical, radiologic and circulating biomarker features. J Immunother Cancer 7:112. https://doi org/10.1186/s40425-019-0583-3

36. Seymour L, Bogaerts J, Perrone A et al (2017) IRECIST: guidelines for response criteria for use in trials testing immunotherapeutics. Lancet Oncol 18:e143-e152. https://doi.org/10.1016/S14702045(17)30074-8

37. Sharma P, Wagner K, Wolchok JD, Allison JP (2011) Novel cancer immunotherapy agents with survival benefit: recent successes and next steps. Nat Rev Cancer 11:805-812. https://doi.org/10.1038/ nrc3153

38. Shields AF, Jacobs PM, Sznol Metal (2018) Immune modulation therapy and imaging: workshop report. J Nucl Med 59:410-417. https://doi.org/10. 2967/jnumed.117.195610

39. Thomas $R$, Somarouthu $B$, Alessandrino $F$ et al (2019) Atypical response patterns in patients treated with Nivolumab. AJR Am J Roentgenol 212:1177-1181. https://doi.org/10.2214/AJR.18. 20938

40. Topalian SL, Hodi FS, Brahmer JRet al (2012) Safety, activity, and immune correlates of anti-PD-1 antibody in cancer. N Engl J Med 366:2443-2454. https://doi.org/10.1056/NEJMoa1200690

41. Travis WD, Costabel U, Hansell DM et al (2013) An official American thoracic society/European respiratory society statement: update of the international multidisciplinary classification of the idiopathic interstitial Pneumonias. Am J Respir Crit Care Med 188:733-748. https://doi.org/10.1164/ rccm.201308-1483ST

42. U.S. Department Of Health And Human Services (2009) Common Terminology Criteria for Adverse Events (CTCAE) Version 4.0

43. Wang GX, Kurra V, Gainor JF et al (2017) Immune checkpoint inhibitor cancer therapy: spectrum of imaging findings. Radiographics 37:2132-2144. https://doi.org/10.1148/rg.2017170085

44. Weber JS, Dummer R, de Pril V et al (2013) Patterns of onset and resolution of immunerelated adverse events of special interest with ipilimumab: Detailed safety analysis from a phase 3 trial in patients with advanced melanoma. Cancer 119:1675-1682. https://doi.org/10.1002/ cncr.27969

45. Wolchok JD, Hamid O, Ribas A et al (2015) Atypical patterns of response in patients (pts) with metastatic melanoma treated with pembrolizumab (MK-3475) in KEYNOTE-001. J Clin Oncol 33:3000-3000. https://doi.org/10.1200/jco.2015. 33.15_suppl.3000

46. Wolchok JD, Hoos A, O'Day Set al (2009) Guidelines for the evaluation of immune therapy activity in solid tumors: immune-related response criteria. Clin Cancer Res 15:7412-7420. https://doi.org/10. 1158/1078-0432.CCR-09-1624

47. Zou W, Chen L (2008) Inhibitory B7-family molecules in the tumour microenvironment. Nat Rev Immunol 8:467-477.https://doi.org/10.1038/ nri2326 